1. Aderka, D., Hausmann, M., Santo, M. et al. Unexplained episodes of fever: an early manifestation of colorectal carcinoma. Israeli J Med Sci 1989, 121: 421-424.

2. Brusch, J.L. \& Wemstein, L. Fever of unknown origin. Med Clin North Am 1988, 72: 1247-1261.

3. Rawlins, M.P., Luff, R.H. \& Cranston, W.I. Pyrexia in renal carcinoma. Lancet 1970, 1: 1371-1373.
4. Saa-Gandi, F.W., Newling, D.W.W. \& Cook, A.M. Pyrexia of unknown origin. An atypical presentation of bladder carcinoma. Br J Urol 1989, 64: 548.

5. Moulonguet, A. \& Camplez, P. Bladder cancer with fever as the first symptom. J Urol Nephrol 1971, 77: 473-476.

\title{
Invasive aspergillosis in two patients with acute lymphoblastic leukaemia in complete remission
}

\author{
Marc L. Turner, Lesley Russell, Leslie J.R. Milne ${ }^{1}$ and Alistair C. Parker \\ Department of Haematology, Royal Infirmary of Edinburgh, Lauriston Place, Edinburgh EH3 9YW and \\ ${ }^{1}$ Mycology Unit, Western General Hospital, Edinburgh, UK
}

Summary: Invasive aspergillosis is a disease of the immunosuppressed patient. We describe two patients with acute lymphoblastic leukaemia who attained complete remission, with partial or complete bone marrow recovery, but who went on to develop fatal invasive aspergillosis contemporaneous with recovery of neutrophil counts. Quantitative recovery of peripheral blood neutrophil counts does not guarantee control of Aspergillus infection, perhaps due to functional neutrophil deficiencies postchemotherapy, and specific defensive strategies adopted by the organism itself.

\section{Introduction}

Aspergillus spp. can cause a spectrum of disease in man, including allergic bronchopulmonary aspergillosis, and formation of aspergilloma in patients with pre-existing upper or lower respiratory tract disease. During neutropenia and other states of immunocompromise, Aspergillus spp. can become invasive, leading to local tissue destruction, haemorrhage, thrombosis, and/or widespread haematogenous dissemination. Invasive aspergillosis poses serious diagnostic and therapeutic problems, and is associated with a high morbidity and mortality.

We describe two patients with acute lymphoblastic leukaemia, who attained complete remission following inductive chemotherapy. These cases are remarkable in that invasive aspergillosis developed contemporaneously with recovery of bone marrow function, and underwent fatal progression despite normal peripheral blood neutrophil counts.

Correspondence: Marc L. Turner, M.R.C.P.

Accepted: 25 September 1992

\section{Case reports}

Case 1

A 51 year old female presented in August 1989 with acute lymphoblastic leukaemia of $\mathrm{T}$ cell lineage. There was no evidence of central nervous system involvement, and she received induction chemotherapy according to UKALL Xa protocol (Table I). She was nursed in a single room without laminar air flow. Complete remission was achieved without incident. In mid-September 1989 she received early intensification (Table I), and during the ensuing period of neutropenia developed oral and oesophageal candidosis, which responded to oral fluconazole.

At the beginning of October 1989 the patient complained of pain around the medial aspect of the left orbit, overlying the left ethmoid sinus. The peripheral blood counts had partially recovered, but over the next 2-3 days she developed left proptosis, with an ipsilateral VIth and sympathetic palsy. The condition progressed to a complete external and internal ophthalmoplegia, and a central field defect. Computed tomography (CT) scan 
Table I MRC UKALL Xa protocol

\section{Induction}

All patients receive the same induction therapy with daunorubicin, vincristine, asparaginase, prednisolone over 1 month, with intrathecal methotrexate Intensification

Two identical blocks of early and late intensification are given, using daunorubicin, vincristine, VP16, thioguanine, cytosine arabinoside and prednisolone, with intrathecal methotrexate

CNS prophylaxis

Cranial irradiation 2.4 Gy with intrathecal methotrexate Maintenance

12 week cycle of daily mercaptopurine, weekly methotrexate, monthly vincristine and prednisolone, to a total of 2 years from time of achieving complete remission.

showed left ethmoid sinus consolidation, but a digital subtraction angiogram failed to confirm cavernous sinus thrombosis. An exploratory ethmoidectomy was undertaken by an external approach, this revealed a small amount of inflammatory material. The specimen was inadvertantly kept at room temperature overnight, and on examination, scanty non-septate hyphae were seen on microscopic examination but no growth was obtained on culture. This observation was taken to indicate contamination and colonization resulting from the delay in storage. However, in view of the clinical signs and symptoms which were consistent with the diagnosis of a zygomycosis, intravenous anti-fungal therapy was started with amphotericin $250 \mu \mathrm{g} / \mathrm{kg} /$ day escalating rapidly to $1.5 \mathrm{mg} / \mathrm{kg} /$ day, and flucytosine $200 \mathrm{mg} / \mathrm{kg} /$ day in divided doses.

Despite these measures, and complete recovery of peripheral blood counts, the disease process showed evidence of progression, with complete loss of vision in the left eye, and episodes of dyscoordination of fine movements of the right hand. A magnetic resonance imaging scan revealed persistent consolidation of the left ethmoid sinus, consolidation of the left sphenoid sinus and retroorbital space, but no evidence of intra-cerebral disease. A re-exploration of the left ethmoid sinus was undertaken in late October, by per-nasal approach. Biopsy specimens proved negative on histological, bacteriological and mycological examination. Anti-fungal treatment was continued.

Four days later the patient developed two wellcircumscribed pulmonary lesions, one in the right mid-zone, and one in the left lung field just posterior to the heart. Two days after this she complained of severe headache, with dyscoordination of the right hand, and truncal ataxia. CT scan revealed evidence of left ethmoid, sphenoid and maxillary sinus consolidation, retro-orbital consolidation, and left cavernous sinus thrombosis. In addition, there were now several low density intra- cerebral lesions. The cerebrospinal fluid revealect neutrophilia 66 cells $/ \mathrm{mm}^{3}$, no lymphoblasts, pro응 tein $1,350 \mathrm{~g} / \mathrm{l}$, glucose $1 \mathrm{mmol} / \mathrm{l}$. Anti-fungal agents were continued. The patient's conscious levels progressively deteriorated over the next 2 weeks. A CT scan revealed a large intra-cerebral haemorr $\overrightarrow{\vec{s}}$ hage, and the patient died 2 days later.

Postmortem examination revealed the acutes lymphoblastic leukaemia to be in complete remis $\overline{-1}$. sion. Examination of the respiratory system confirmed two areas of discrete consolidation aso noted radiologically, culture yielded Aspergillus fumigatus. Examination of the head and neck $\vec{\circ}$ revealed an inflammatory exudate involving the left ethmoid, sphenoid and maxillary sinuses, and the left retro-orbital space. Both cavernous sinusesg showed inflammation, with involvement of the cranial nerves on the left. The left internal carotido was totally occluded by thrombus, associated with ${ }_{\infty}^{\circ}$ fungal invasion of part of its wall, and there wasextensive infarction of the left cerebral hemisphere There was more limited infarction of the rightor cerebral hemisphere, with intracerebral haemorr-윽 hage, presumably due to a mycotic aneurysm.There was a widespread basal meningitis associated with fungal infiltration of the subarachnoid vessels $: 2$

Branched septate hyphae were seen in the prepared sections. The overall appearance vagse consistent with the diagnosis of aspergillosis. The was no evidence of non-septate hyphae that wotido have indicated significant involvement of $*$ a zygomycote.

\section{Case 2}

A 22 year old male presented in October 1989 with a 10 day history of generalized illness. Clinica examination was unremarkable, but the periphera? blood demonstrated pancytopenia. The bone mar-o row aspirate revealed normal overall marrow cel lularity, with a patchy increase of lymphocytes and ${ }_{3}^{3}$ plasma cells. The patient was initially managed with supportive measures, including red cell trans- -3 fusion and broad spectrum antibiotics to cover $\mathrm{a}_{\circ}$ pyrexia of unknown origin. His condition improved, and the pancytopenia resolved spon $\Rightarrow \frac{7}{O}$ taneously. The patient was discharged at the end of October, and remained well over the next 2 months, $N$ with a persisting mild neutropenia but otherwise normal peripheral blood counts.

At the beginning of January 1990 the patient again deteriorated with development of pan $\times$ cytopenia. The patient was diagnosed as suffering from acute lymphoblastic leukaemia on the basis of bone marrow morphology and immunophenotyp-ing, and commenced on induction chemotherapy $\frac{0}{0}$ according to UKALL Xa protocol (Table I). He was nursed in a single room without laminar air $\frac{?}{\mathbb{C}}$ flow. 
The patient was pyrexial for 24-48 hours around the start of chemotherapy, clinical examination, bacteriological and mycological cultures were negative, and ceftazidime and netilmicin were commenced. The patient was well until day 15 , when he developed pleuritic chest pain at the right lung apex; clinical examination was unremarkable, as was the chest radiograph. No sputum was produced at any stage. A Pseudomonas sp. was identified from one blood culture, mycological cultures remained negative, and azlocillin was added to the antibiotic regime. On day 18 the chest pain persisted, the patient remained apyrexial and the clinical examination was unremarkable. The chest X-ray revealed evidence of right apical consolidation. Over the next 7 days, the pain settled, clinical examination remained normal, and the peripheral blood counts returned to normal. The right apical consolidation persisted, but on day 27 cavitation occurred. On day 30 the patient suffered sudden massive haemoptysis and died.

Postmortem examination revealed a large mycotic abcess at the apex of the right lung, lined by necrotic material, inflammatory exudate, and hyphae consistent with Aspergillus spp. There was erosion of at least one major branch of the pulmonary artery, with extensive haemorrhage within both the right pleural sack, and the bronchial tree. The remainder of the postmortem examination was remarkably normal, no other mycological investigation was carried out. The bone marrow revealed complete remission of the acute lymphoblastic leukaemia.

\section{Discussion}

Invasive aspergillosis is primarily a disease of the immune-suppressed patient. ${ }^{1}$ Recognized high-risk groups include bone marrow, renal, liver and heart transplant recipients, ${ }^{2}$ and patients undergoing induction chemotherapy for acute leukaemia ${ }^{3}$ and other haematological malignancies. The disease has also been described in patients with diabetes mellitus, burns, chronic granulomatous disease, and acquired immunodeficiency syndrome. ${ }^{4}$ Occasional cases have been described in patients with normal immune function. ${ }^{5}$

Aspergillus secretes an acid protease which allows it to breach most natural barriers, including cartilage and bone. It displays marked angiotropism; vascular invasion can lead to haemorrhage, thrombosis or haematogenous dissemination.

Studies in man, ${ }^{6}$ and in experimental animal systems $^{7,8}$ have emphasized the predominant importance of prolonged neutropenia, neutrophil dysfunction and prolonged treatment with corticosteroids as factors predisposing to tissue invasion. Patients with acute lymphoblastic leukaemia undergoing current standard intensive chemotherapy regimes experience periods of neutropenia lasting at least $10-14$ days, and receive up to 6 weeks of high-dose steroids.

A prominent feature of the two cases we describe is the appearance and progression of tissue invasion contemporaneous with the recovery of peripheral blood neutrophil counts. Clinical studies on patients following allogeneic bone marrow transplantation have shown that, although neutrophil counts usually recover within $2-3$ weeks of the transplant procedure, neutrophil function - as reflected by chemotaxis, superoxide production, and phagocytic bactericidal activity can be defective for several months. ${ }^{9}$ It is unknown whether patients receiving standard intensive chemotherapy are similarly affected.

There is experimental evidence to suggest that Aspergillus conidia in resting phase is relatively resistant to neutrophil killing due to suboptimal stimulation of neutrophil migration, phagocytosis and degranulation, ${ }^{10}$ and that the organism may achieve this by opsonization with heat-labile serum components. ${ }^{11}$ In addition, Aspergillus is a constitutive producer of catalase, an enzyme which destroys superoxide anion and hydrogen peroxide, and may attenuate neutrophil generation of highly reactive chlorinated oxidants in phagocytic vesicles and at the extracellular aspect of the plasma membrane. $^{12}$

We feel that in these patients the prolonged periods of neutropenia and steroids were sufficient to allow aspergillus infection to become established, whilst neutrophil function on marrow recovery may have been insufficient to control the infection. Indeed, the development of a tissue inflammatory reaction with liquefaction of damaged tissue may, in this situation, paradoxically augment fungal invasion and dissemination. ${ }^{12}$

In summary, although aspergillus infection is often considered in transplant recipients, it should not be forgotten as an important cause of disease in other groups. In those patients undergoing routine intensive chemotherapy for acute leukaemia, quantitative recovery of peripheral blood neutrophil counts does not assure control of aspergillus infection, and indeed the disease may progresss despite marrow recovery and intravenous anti-fungal agents.

We feel that the effects of routine chemotherapy regimes on neutrophil function, the defensive strategies adopted by Aspergillus, and the potentially adversive affects of neutrophil recovery on areas of existing tissue damage are all areas which would repay further study.

\section{Acknowledgements}

Our thanks to the Kay Kendall Leukaemia Trust and the Melville Trust for the Care and Cure of Cancer for financial support. 
References

1. Stein, D.K. \& Sugar, A.M. Fungal infections in the immunocompromised host. Diagn Microbiol Infect Dis 1989, 12: $221 \mathrm{~S}-228 \mathrm{~S}$.

2. Denning, D.W. \& Stevens, D.A. Antifungal and surgical treatment of invasive aspergillosis: review of 2,121 published cases. Rev Infect Dis 1990, 12: 1147-1201.

3. Klimowski, L.L., Rotstein, C. \& Cummings, K.M. Incidence of nosocomial aspergillosis in patients with leukaemia over a twenty year period. Infect Control Hosp Epidemiol 1989, 10: 299-305.

4. Denning, D.W., Follansbee, S.E., Scolaro, M., Norris, S., Edelstein, H. \& Stevens, D.A. Pulmonary aspergillosis in the acquired immunodeficiency syndrome. N Engl J Med 1991, 324: 654-662.

5. Cook, D.J., Achong, M.R. \& King, D.E. Disseminated aspergillosis in an apparently healthy patient. Am J Med 1990, 88: 74-76.

6. Bodey, G.P. \& Vartivarian, S. Aspergillosis. Eur J Clin Microbiol Infect Dis 1989, 8: 413-437.
7. Eisenstein, D.J., Biddinger, P.W. \& Rhodes, J.C. Experimental murine invasive pulmonary aspergillosis. Am J Clin Path 1990, 93: 510-515.

8. Spreadbury, C.L., Krausz, T., Pervez, S. \& Cohen, J. Invasive $C$ aspergillosis: clinical and pathological features of a new animal model. J Med Vet Mycol 1989, 27: 5-15.

9. Zimmerci, W., Zarth, A., Gratwohl, A. \& Speck, B. Neutro-으 phil function and pyogenic infection in bone marrow trans $-\bar{O}$ plant recipients. Blood 1991, 77: 393-399.

10. Robertson, M.D., Seaton, A. \& Raeburn, J.A. Phagocytic cell responses to Aspergillus fumigatus. FEMS Microbioß口 Immunol 1989, 47: 305-306.

11. Robertson, M.D., Kerr, K.M. \& Seaton, A. Killing of aspergillus fumigatus spores by human lung macrophages, $\mathrm{aO}$ paradoxical effect of heat labile serum components. $J \mathrm{Med}-$ Vet Mycol 1989, 27: 295-302.

12. Weiss, S.J. Tissue destruction by neutrophils. $N$ Engl J Med 1989, 320: $365-376$.

\title{
Parathyroid crisis and acute viral hepatitis B infection
}

\author{
Hugh E. Mulcahy, Frank E. Murray, Rosemarie Freaney ${ }^{1}$ and \\ John E. Hegarty
}

Gastroenterology and Liver Unit and ${ }^{1}$ Metabolic Unit, St Vincent's Hospital, Elm Park, Dublin 4, Irelan

\begin{abstract}
Summary: The simultaneous development of acute hyperparathyroidism and viral hepatitis B infection in a 72 year old male is described. Resolution of the hepatitis was accompanied by improvement in the parathyroid hormone mediated hypercalcaemia. It is postulated that antibodies to the hepatitis B virus may have altered the calcium 'set point' allowing uncontrolled synthesis and release of parathyroid hormone during the acute illness.
\end{abstract}

\section{Introduction}

Hypercalcaemia, a rarely recognized complication of acute viral hepatitis has been reported in association with acute non-A non-B hepatitis and attributed to virus-induced cytokine production. ${ }^{1}$ Acute primary hyperparathyroidism (parathyroid crisis) has been described as an unusual form of hyperparathyroidism characterized by life-threatening hypercalcaemia ${ }^{2}$ and has not previously been reported in association with viral hepatitis.

\section{Case report}

A 71 year old man was admitted with a 3 week history of anorexia, nausea, vomiting, malaise,

Correspondence: H. Mulcahy, M.B., M.R.C.P.I.

Accepted: 2 November 1992 fatigue, arthralgia, myalgia and polyuria. A benign gastric ulcer and reflux oesophagitis, confirmed? endoscopically, had been diagnosed 9 months previously and successfully treated with ranitidine? $150 \mathrm{mg}$ twice daily for 8 weeks. There was no history of transfusion with blood or blood products, homosexual contact, alcohol abuse, hep- N atotoxic drug ingestion, recent foreign travel, ingestion of antacids, lithium, thiazides, vitamin $D_{N}$ or calcium-containing preparations. At presenta- $\omega$ tion physical examination revealed jaundice and hepatomegaly in the absence of stigmata of chronico liver disease.

\section{Results}

Investigations revealed a serum albumin of $33 \mathrm{~g} / 1 \stackrel{\mathrm{P}}{\mathrm{P}}$ (normal range: $35-50$ ), bilirubin $85 \mathrm{mmol} / 10$ 